$10.1117 / 2.1201608 .006615$

\title{
Terahertz imaging of biomechanical interfaces
}

\author{
Erwin Hack, Lorenzo Valzania, and Peter Zolliker
}

A terahertz laser, combined with an uncooled microbolometer camera, is used in a digital holography method to provide improved resolution of skin profiles.

In addition to being fashionable, textile materials for clothing need to be comfortable, functional, and biocompatible. These requirements are especially important for cases where skin is in continuous contact with textiles, e.g., for long-distance hikers, babies in wet diapers, or bedridden patients. Such cases can pose a risk to health, especially when sweat, pressure, and friction factors are considered. In these situations, it is essential to determine the physical parameters involved, such as the real contact area of the skin-textile interface and the distribution of water at this interface. Knowledge of these parameters is required for gaining a deeper understanding of processes, such as impairment of the physiological functions of skin caused by swelling or loss of blood circulation, as well as for the development of improved materials. It is challenging, however, to study the skintextile interface because it is hidden by the material. Furthermore, the insertion of a sensor at the interface would drastically alter the local physical properties.

To overcome this inaccessible skin-textile interface problem, terahertz radiation can be employed. ${ }^{1}$ Terahertz waves are electromagnetic waves with frequencies between 0.3 and $3 \mathrm{THz}$ (corresponding to wavelengths of $100-1000 \mu \mathrm{m}$ ). The advantage of using terahertz waves is that they can penetrate non-conductive materials (such as textiles), they are sensitive to humidity, and they are reflected from skin surfaces and other interfaces. ${ }^{2}$ Moreover, owing to their long wavelength, terahertz waves are unaffected by vibrations and surface roughness. However, this is at the expense of imaging resolution.

In our work, we have therefore developed a terahertz imaging technique with which we can achieve enhanced resolution. In our method, we use coherent radiation from a terahertz laser to provide additional phase information, which we obtain using digital holography. ${ }^{3}$ We measure the surface profile of the skin and use this information to calculate the real contact area

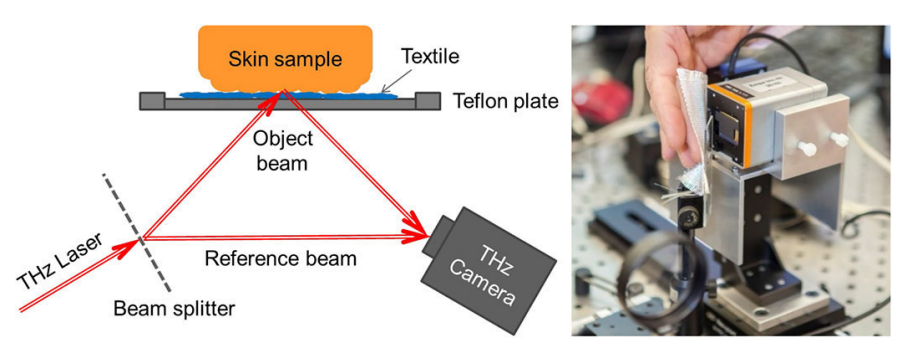

Figure 1. Left: Schematic diagram of the proposed terahertz (THz) laser system for imaging a skin-textile interface. Right: Photograph of the hologram recording system, with a human hand positioned for imaging of a fingertip.

of the skin-textile interface. This parameter can then be used in the adhesion friction model (in which the frictional behavior of skin is dominated by adhesion), in combination with a pressuredependent term, to model the coefficient of friction. ${ }^{4,5}$

In our imaging procedure (see Figure 1), a terahertz beam from a high-power far-IR laser is split into an object beam and a reference beam. The object beam is used to illuminate a skin sample that is placed behind a plate. We use the reference beam to recover the phase of the object beam via holographic interference on a terahertz detector plane. We use an uncooled microbolometer camera with a resolution of $480 \times 640$ pixels (at a pitch of $17 \mu \mathrm{m}$ ) to record the interference pattern that is generated. Although this camera was designed for thermal imaging, it has also been found to be suitable for digital off-axis terahertz holography. ${ }^{6}$ Digital holography provides several advantages over imaging with a lens (e.g., compact experimental apparatus, high lateral resolution, and the ability to carry out numerical reconstructions on planes that are not parallel to the detector plane). In our setup, the amplitude and phase of the object beam are reconstructed at a distance of $9 \mathrm{~mm}$ from the detector plane and at an angle of $45^{\circ}$, with respect to the detector plane.

We have previously employed terahertz holography (in transmission) and confirmed that the lateral resolution was 

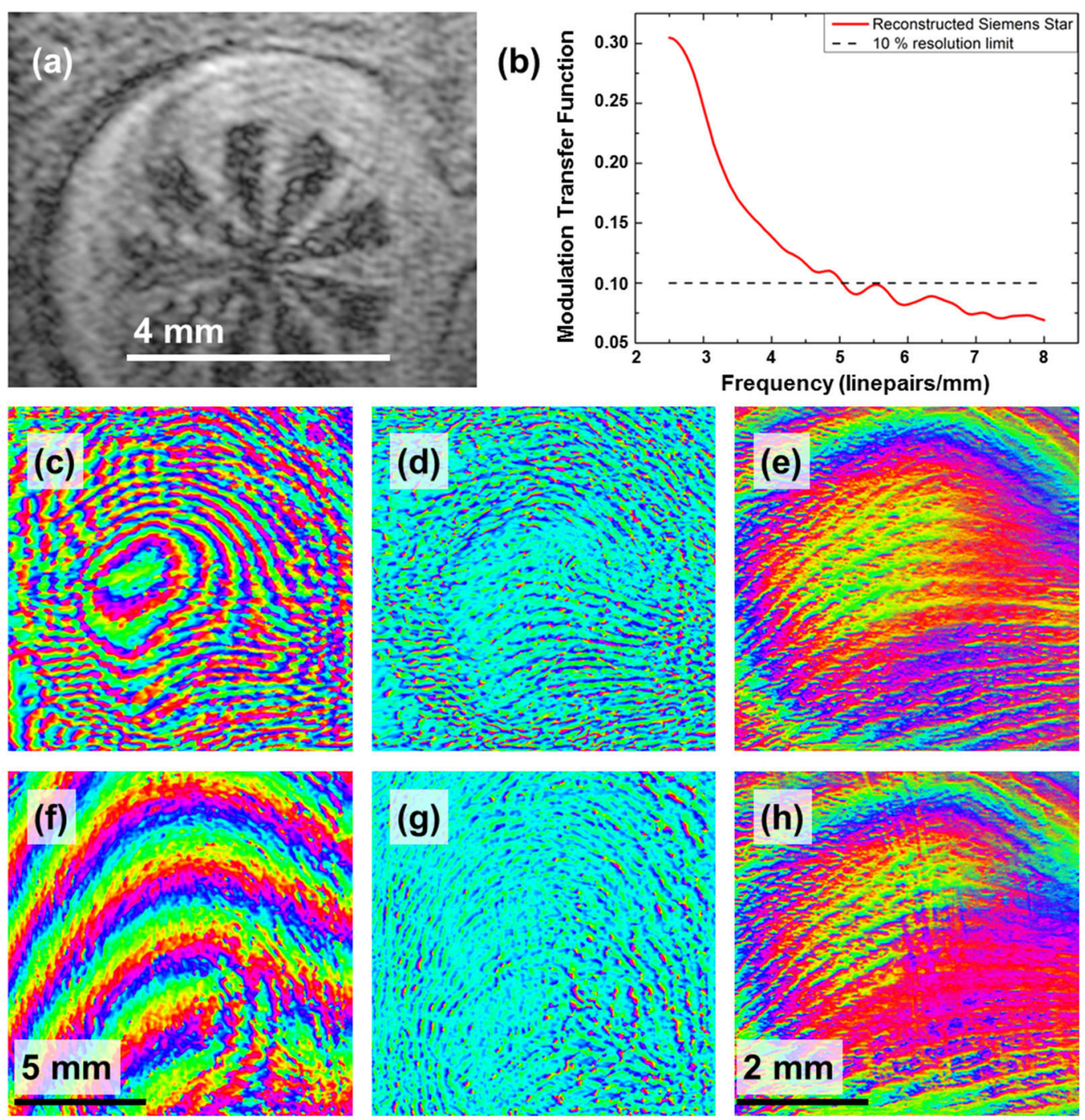

Figure 2. (a) Reconstructed image of a Siemens star obtained using our terahertz holography technique. (b) Lateral resolution of our terahertz holography technique (in line pairs/mm), measured by modulation transfer. (c) Reconstructed wrapped phase of a fingertip replica profile, obtained by terahertz holography. (d) High-frequency component of the image in (c), obtained by high-pass filtering. (e) Reconstructed wrapped phase of the fingertip profile, obtained by optical fringe projection. (f) Image as in (c), but where the fingertip was placed behind a Teflon plate for imaging. ( $g$ ) High-frequency component of the image in (f), obtained by high-pass filtering. (h) Image as in (e), but where the fingertip was placed behind a quartz glass plate for imaging. The wrapped phases are displayed as periodic color maps, in which the period corresponds to differences in optical path length of the terahertz beam wavelength.

better than $200 \mu \mathrm{m}$ (just sufficient for resolving skin furrows). ${ }^{7}$ To estimate the lateral resolution of our technique (in reflection), we imaged a metallic Siemens star, as shown in Figure 2(a). We found-see Figure 2(b) - that the resolution reached 5 line pairs $/ \mathrm{mm}$ at a modulation transfer function of $10 \%$, which we take to be the resolution limit.

To benchmark the resolution of our technique, in both the lateral direction and the profile height, we have also conducted imaging of a fingertip replica. The reconstructed wrapped phase of this image is shown in Figure 2(c), where the period of the colored bands (from red to blue) corresponds to differences in optical path length of the terahertz beam wavelength. At the resolution limit of our technique (i.e., 5 line pairs $/ \mathrm{mm}$ ), we were able to image the profile of the fingertip replica in reflection.

Continued on next page 
We used high-pass filtering-see Figure 2(d) - to enhance the sub-millimeter ripple structure. Low-frequency phase distortions introduced by a $1 \mathrm{~mm}$-thick opaque Teflon plate in front of the fingertip can clearly be seen in Figure 2(f). The highfrequency components of the image in Figure 2(f), which represent the fingertip, are shown in Figure 2(g). For comparison, we also obtained images by white-light fringe projection. The image of the fingertip replica obtained in this way is shown in Figure 2(e), and the image of the fingertip behind a transparent quartz glass plate (used instead of the Teflon plate) is shown in Figure 2(h).

In summary, we have devised an apparatus for highresolution off-axis digital terahertz holography and have shown its potential for the imaging of hidden biomechanical interfaces. To investigate the parameters involved in skin irritation, in future experiments we intend to insert a textile patch between a Teflon plate and a skin sample. The textile will thus act as a diffractive structure that adds complexity to the object wave reflected by the skin surface. We are confident that we will be able to separate the contributions of the Teflon plate and textile patch in the hologram by using different recording angles and by varying the wavelength of the incident terahertz beam.

We acknowledge funding by the Swiss National Science Foundation (contract 200021_160078/1).

\section{Author Information}

\section{Erwin Hack, Lorenzo Valzania, and Peter Zolliker}

Swiss Federal Laboratories for Materials Science and Technology (Empa)

Dübendorf, Switzerland

Erwin Hack is a senior scientist at Empa. He holds a diploma in theoretical physics and a PhD in physical chemistry from the University of Zurich, Switzerland. His research interests include terahertz imaging, speckle pattern interferometry, and the validation of numerical models using full-field techniques. He is a lecturer at the Swiss Federal Institute of Technology, Zurich (ETHZ).

Lorenzo Valzania is a second-year PhD student. He is working on terahertz imaging of biomechanical interfaces via digital holography. He holds a master's degree in engineering physics from the Polytechnic University of Milan, Italy. His research interests include full-field optical measurement techniques, terahertz radiation for non-destructive testing, and x-ray tomography.
Peter Zolliker obtained a degree in physics from ETHZ and a $\mathrm{PhD}$ in crystallography from the University of Geneva, Switzerland. He was a postdoctoral fellow at the Brookhaven National Laboratory before joining the research and development team at Gretag Imaging. In 2003, he joined Empa to carry out research into imaging, image quality, and psychophysics. His current research focuses are in terahertz imaging, holography, and spectroscopy.

References

1. X. C. Zhang and J. Xu, THz technology in nondestructive evaluation, Introduction to THz Wave Photonics, pp. 175-199, Springer, 2010.

2. D. K. George and A. G. Markelz, Terahertz spectroscopy of liquids and biomolecules, in K.-E. Peiponen, A. Zeitler, and M. Kuwata-Gonokami (eds.), Terahertz Spectroscopy and Imaging, pp. 229-250, Springer, 2013.

3. P. Zolliker and E. Hack, THz holography in reflection using a high resolution microbolometer array, Opt. Express 23, pp. 10957-10967, 2015.

4. S. Derler, G. M. Rotaru, W. Ke, L. El Issawi-Frischknecht, P. Kellenberger, A. Scheel-Sailer, and R. M. Rossi, Microscopic contact area and friction between medical textiles and skin, J. Mechan. Behav. Biomed. Mater. 38, pp. 114-125, 2014.

5. F. P. Bowden and D. Tabor, Adhesion between solid surfaces: the influence of liq uid films, The Friction and Lubrication of Solids, pp. 299-314, Oxford University Press, 1986

6. E. Hack, L. Valzania, G. Gäumann, M. Shalaby, C. P. Hauri, and P. Zolliker, Comparison of thermal detector arrays for off-axis $\mathrm{THz}$ holography and real-time $\mathrm{THz}$ imaging, Sensors 16, p. 221, 2016. doi:10.3390/s16020221

7. E. Hack and P. Zolliker, High-resolution terahertz holography for profilometry in transmission, PhotoMechanics Conf., pp. 116-118, 2015. 